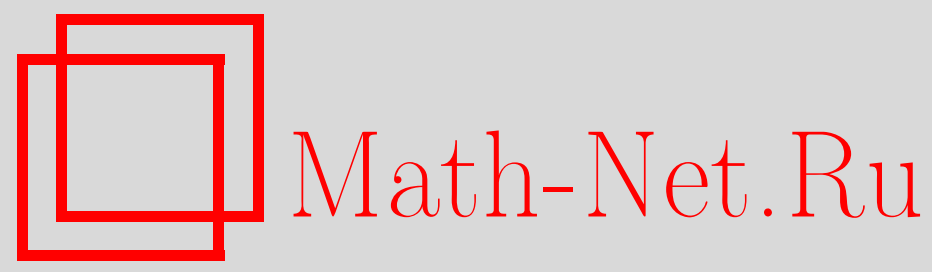

А. С. Кузьмина, Описание конечных нильпотентных колец, имеющих планарные графы делителей нуля, Дискрет. матем., 2009, том 21, выпуск 4, 60-75

DOI: https://doi.org/10.4213/dm1071

Использование Общероссийского математического портала Math-Net.Ru подразумевает, что вы прочитали и согласны с пользовательским соглашением http://www . mathnet.ru/rus/agreement

Параметры загрузки:

IP: 35.173 .137 .237

26 апреля 2023 г., $15: 26: 21$ 


\title{
Описание конечных нильпотентных колец, имеющих планарные графы делителей нуля
}

\author{
() 2009 г. А. С. Кузьмина
}

\begin{abstract}
Графом делителей нуля ассоциативного кольца $R$ называется граф, вершинами которого являются все ненулевые (односторонние и двусторонние) делители нуля кольца, причем две различные вершины $x, y$ соединяются ребром тогда и только тогда, когда $x y=0$ или $y x=0$. В работе полностью описаны конечные ненильпотентные кольца, имеющие планарные графы делителей нуля. Ранее автором и Ю. Н. Мальцевым были исследованы нильпотентные конечные кольца, графы делителей нуля которых планарны. Таким образом, настоящая работа завершает описание конечных колец с планарными графами делителей нуля.
\end{abstract}

В данной работе рассматриваются ассоциативные кольца (не обязательно коммутативные и не обязательно имеющие единицу).

Определение 1. Графом делителей нуля кольца $R$ называется граф, вершинами которого являются все ненулевые делители нуля кольца (односторонние и двусторонние), причем две различные вершины $x, y$ соединяются ребром тогда и только тогда, когда $x y=0$ или $y x=0$.

Обычно граф делителей нуля кольца $R$ обозначается через $\Gamma(R)$. Мы также будем использовать это обозначение.

Понятие графа делителей нуля было введено в работе [1]. И. Бек ввел это понятие для коммутативного кольца и вершинами графа делителей нуля считал все элементы кольца. В статье [2] определение было изменено: в качестве вершин графа делителей нуля коммутативного кольца авторы этой работы рассматривали лишь ненулевые делители нуля. С этого момента теория графов делителей нуля стала достаточно интенсивно развиваться. Кроме того, понятие графа делителей нуля было распространено и на некоммутативный случай (см., например, [3]).

Одним из направлений исследований в этой области стало описание колец, граф делителей нуля которых удовлетворяет определенному условию. Так, в работах $[4,5]$ исследуются коммутативные конечные кольца с единицей, графы делителей нуля которых планарны. В частности, в [4] приведено полное описание конечных коммутативных разложимых колец с единицей, у которых графы делителей нуля планарны, а в [5] составлен полный список конечных коммутативных неразложимых колец с единицей, имеющих планарные графы делителей нуля. В [6] полностью описаны конечные нильпотентные (не обязательно коммутативные) кольца, имеющие планарные графы делителей нуля.

В данной работе мы приводим полное описание конечных ненильпотентных (не обязательно коммутативных) колец с планарными графами делителей нуля. Таким образом, 
результаты данной работы в объединении с результатами статьи [6] дают полный список конечных колец, чьи графы делителей нуля планарны.

Введем обозначения, используемые в настоящей работе.

Запись $R=A+B$ означает, что аддитивная группа кольца $R$ разлагается в прямую сумму двух своих подгрупп $A$ и $B$. Через $\langle a\rangle$ мы будем обозначать аддитивную подгруппу аддитивной группы кольца $R$, порожденную элементом $a \in R$.

Пусть для любого простого числа $p$

$$
\begin{aligned}
& N_{0, p^{n}}=\langle a\rangle, \quad p^{n} a=0, \quad a^{2}=0 ; \\
& N_{p^{2}}=\langle a\rangle, \quad p^{2} a=0, \quad a^{2}=p a ; \\
& N_{p, p}=\langle c\rangle \dot{+}\langle d\rangle, \quad p c=p d=0, \quad c^{2}=d, \quad c d=d c=d^{2}=0 ;
\end{aligned}
$$

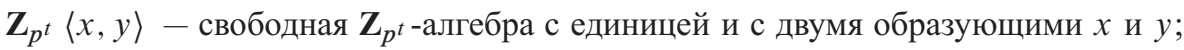
$M_{n}(R)-$ полное кольцо матриц размера $n \times n$ над кольцом $R$;

$$
\begin{aligned}
& A_{p^{n}}=\left(\begin{array}{cc}
G F\left(p^{n}\right) & G F\left(p^{n}\right) \\
0 & 0
\end{array}\right) ; \quad A_{p^{n}}^{0}=\left(\begin{array}{cc}
G F\left(p^{n}\right) & 0 \\
G F\left(p^{n}\right) & 0
\end{array}\right) ; \quad B_{p}=\left(\begin{array}{cc}
G F(p) & G F(p) \\
0 & G F(p)
\end{array}\right) ; \\
& C_{p^{n}}=\left\{\left(\begin{array}{cc}
a & b \\
0 & \sigma(a)
\end{array}\right) ; a, b \in G F\left(p^{n}\right), \sigma \text { автоморфизм поля } G F\left(p^{n}\right), \sigma \neq 1\right\} .
\end{aligned}
$$

Заметим, что $C_{p^{n}}$ является подкольцом кольца $M_{2}\left(G F\left(p^{n}\right)\right)$, то есть операции в кольце $C_{p^{n}}$ суть обычные операции сложения и умножения матриц.

Пусть $R$ - произвольная $\mathbf{Z}_{p}$-алгебра и $R^{\#}-$ множество $\mathbf{Z}_{p} \times R$ с введенными на нем операциями

$$
\begin{aligned}
(m, a)+(n, b) & =(m+n, a+b), \\
(m, a) \cdot(n, b) & =(m n, m b+n a+a b)
\end{aligned}
$$

для любых $m, n \in \mathbf{Z}_{p}, a, b \in R$. Множество $R^{\#}$ с определенными таким образом операциями сложения и умножения является $\mathbf{Z}_{p}$-алгеброй с единицей.

Пусть аддитивная группа кольца $R$ разлагается в прямую сумму своих аддитивных подгрупп $A_{i}, i=1, \ldots, n$, и $n \geqslant 2$, то есть $R=A_{1} \dot{+} \ldots \dot{+} A_{n}$. Если все $A_{i}$ являются двусторонними идеалами кольца $R$, то кольцо $R$ называют разложимым и пишут $R=A_{1} \oplus \ldots \oplus A_{n}$. Соответственно кольцо называется неразложимым, если оно не является разложимым.

Пусть $R=A_{1} \oplus \ldots \oplus A_{n}, n \geqslant 2$. Поскольку $A_{i}$ являются двусторонними идеалами кольца $R$, справедливо включение $A_{i} A_{j} \subseteq A_{i} \cap A_{j}=\{0\}$ при $i \neq j$, то есть $A_{i} A_{j}=0$ при $i \neq j$. Обратно, пусть $R=A_{1} \dot{+} \ldots \dot{+} A_{n}$ (прямая сумма аддитивных групп) и $A_{i} A_{j}=\{0\}$ при $i \neq j$. Тогда все $A_{i}$ являются двусторонними идеалами кольца $R$.

Далее через $J(R)$ мы будем обозначать радикал Джекобсона кольца $R$ (см. с. 73 в [9]).

Конечное кольцо с единицей называется локальным, если $R / J(R)$ является полем (см. [9]).

Пусть $R$ - произвольное кольцо (не обязательно содержащее единицу) и $f$ - идемпотент кольца $R$, то есть $f^{2}=f$. Введем обозначения

$$
\begin{aligned}
f R f & =\{f r f ; r \in R\}, \\
f R(1-f) & =\{f r-f r f ; r \in R\}, \\
(1-f) R f & =\{r f-f r f ; r \in R\}, \\
(1-f) R(1-f) & =\{r-f r-r f+f r f ; r \in R\} .
\end{aligned}
$$


Известно, что справедливо следующее разложение, называемое двусторонним пирсовским разложением кольца $R$ (см. с. 32 в [9]):

$$
R=f R f \dot{+} f R(1-f) \dot{+}(1-f) R f \dot{+}(1-f) R(1-f) .
$$

Приведем некоторые определения из теории графов, используемые в настоящей работе (см. [7]).

Двудольный граф $G$ - это граф, множество вершин $V$ которого можно разбить на два непересекающихся непустых подмножества $V_{1}$ и $V_{2}$ таким образом, что каждое ребро графа $G$ соединяет вершины из разных подмножеств. Если двудольный граф $G$ содержит все ребра, соединяющие множества $V_{1}$ и $V_{2}$, то этот граф называется полным двудольным графом. Полные двудольные графы обозначаются $K_{n, m}$, где $n=\left|V_{1}\right|$ и $m=\left|V_{2}\right|$. Полный двудольный граф $K_{1, n}$ называется звездой. Полным $n$-вершинным графом $K_{n}$ называется граф (без петель и кратных ребер), все $n$ вершин которого смежны между собой. Граф называется планарным, если его можно изобразить на плоскости так, чтобы никакие два его ребра не пересекались. Два графа называются гомеоморфными, если их можно получить из одного и того же графа с помощью включений в его ребра новых вершин степени 2.

Напомним также, что граф планарен тогда и только тогда, когда он не содержит подграфа, гомеоморфного $K_{3,3}$ и $K_{5}$ (теорема Куратовского, [7]). На протяжении данной статьи мы неоднократно будем пользоваться этой теоремой.

Предложение 1. Пусть $R$ - конечное неразложимое ненильпотентное кольцо с планарным графом делителей нуля. Тогда $R$ удовлетворяет одному из следующих условий:

(1) $R \cong G F\left(p^{n}\right)$;

(2) $R \cong M_{2}(G F(2))$;

(3) $R \cong B_{2}$;

(4) $R / J(R)=G F(q)$, причем $J(R) \neq(0) u|R| \leqslant 27$.

Доказательство. Пусть $R$ - конечное неразложимое ненильпотентное кольцо с планарным графом делителей нуля. Рассмотрим сначала случай, когда $R-$ полупростое кольцо, то есть $J(R)=(0)$. Тогда $R=M_{r}(G F(q))$. При $r \geqslant 3$ граф делителей нуля множества $\left\{e_{13}, e_{23}, e_{33}\right\} \cup\left\{e_{11}, e_{12}, e_{22}\right\}$, где $e_{i j}-$ матричные единицы, содержит $K_{3,3}$; получаем противоречие. Следовательно, $r \leqslant 2$. Если $r=2$ и $q \geqslant 3$, то для любого элемента $\alpha \in G F(q) \backslash\{0,1\}$ множество $\left\{e_{12}, e_{11}, \alpha e_{11}\right\} \cup\left\{\alpha e_{12}, e_{22}, \alpha e_{22}\right\}$ образует $K_{3,3}$; получаем противоречие. Итак, $R \cong G F\left(p^{n}\right)$ или $R \cong M_{2}(G F(2))$. Как видно из рис. 1 , граф $\Gamma\left(M_{2}(G F(2))\right)$ планарен.

Пусть $J(R) \neq(0)$ и

$$
\bar{R}=R / J(R) \cong \bigoplus_{i=1}^{m} M_{n_{i}}\left(G F\left(q_{i}\right)\right)
$$

Далее, пусть $e_{1}, \ldots, e_{m}-$ множество ортогональных идемпотентов кольца $R$ таких, что $\bar{e}_{i}$ является единицей в $M_{n_{i}}\left(G F\left(q_{i}\right)\right), i=1, \ldots, m$. Введем обозначение

$$
e=\sum_{i=1}^{m} e_{i}
$$




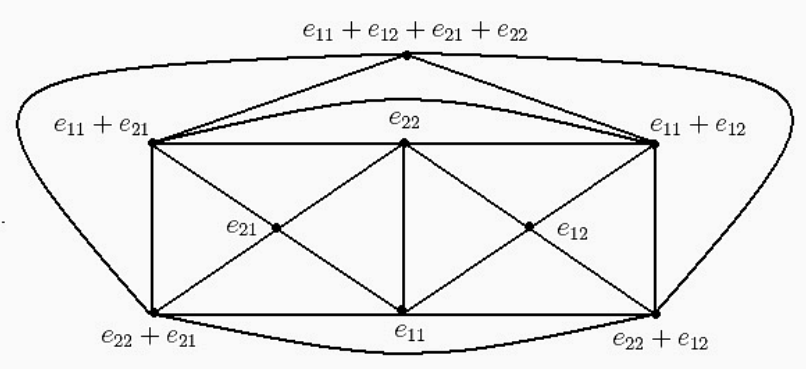

Рис. 1. Граф $\Gamma\left(M_{2}(G F(2))\right)$

В частности, если $R$ имеет единицу 1 , то $e=1$ (см. с. 85 в [8]). Рассмотрим подкольцо с единицей

$$
e R e=\sum_{i, j=1}^{m} e_{i} R e_{j}
$$

Введем обозначение $R_{i}=e_{i} R e_{i}, i=1, \ldots, m$. Тогда

$$
R_{i} / J\left(R_{i}\right) \cong \bar{e}_{i} \bar{R} \bar{e}_{i}=M_{n_{i}}\left(G F\left(q_{i}\right)\right)
$$

(см. с. 75 в [9]). Поскольку $R_{i}$ - кольцо с единицей, согласно [8], найдется такое локальное кольцо $S_{i}$, что

$$
R_{i} \cong M_{n_{i}}\left(S_{i}\right), \quad S_{i} / J\left(S_{i}\right) \cong G F\left(q_{i}\right)
$$

Таким образом,

$$
e R e=\sum_{i=1}^{m} M_{n_{i}}\left(S_{i}\right)+\sum_{i \neq j} e_{i} R e_{j}
$$

Согласно [9]

$$
R=e R e \dot{+} e R(1-e) \dot{+}(1-e) R e \dot{+}(1-e) R(1-e) .
$$

Если $m \geqslant 4$, то элементы $\left\{e_{1}, e_{1}+e_{2}, e_{2}\right\} \cup\left\{e_{3}, e_{3}+e_{4}, e_{4}\right\}$ образуют $K_{3,3}$; получаем противоречие. Следовательно, $m \leqslant 3$. Рассмотрим отдельно каждый случай в зависимости от значения, которое принимает $m$.

Случай 1. $m=3$. Предположим, что найдется ненулевой элемент $a \in e R(1-e)$. Тогда $K_{3,3}$ содержится в $\Gamma(R)$. В самом деле, граф делителей нуля множества $\left\{e_{1}, e_{1}+e_{2}, e_{2}\right\} \cup\left\{a, e_{3}, a+e_{3}\right\}$ содержит в качестве подграфа $K_{3,3}$; получаем противоречие. Следовательно, $e R(1-e)=(0)$. Аналогично находим, что $(1-e) R e=(1-e) R(1-e)=(0)$. Отсюда $R=e R e$. Предположим, что для некоторых $i, j, i \neq j$, существует ненулевой элемент $a \in e_{i} R e_{j}$. Тогда множество $\left\{e_{j}, e_{k}+e_{j}, e_{k}\right\} \cup\left\{e_{i}, a, a+e_{i}\right\}$, где $k \neq i, j$, образует граф $K_{3,3}$; получаем противоречие. Поэтому $e_{i} R e_{j}=(0)$ для всех $i, j(i \neq j)$. Значит, $R=\bigoplus_{i=1}^{3} R_{i}$, что противоречит неразложимости кольца $R$. 
Случай 2. $m=2$. Если $\left|R_{1}\right| \geqslant 4$ и $\left|R_{2}\right| \geqslant 4$, то $\Gamma(R)$ содержит в качестве подграфа граф $K_{3,3}$. Поэтому, например, $\left|R_{1}\right| \leqslant 3$. Следовательно, $R_{1} \cong G F(p)$, где $p=2,3$. Как отмечалось выше, $R_{2} \cong M_{n_{2}}\left(S_{2}\right)$, причем $S_{2} / J\left(S_{2}\right) \cong G F\left(p^{n}\right)$. Рассуждая так же, как это было сделано для полупростого случая в самом начале доказательства предложения, легко доказать, что $R_{2} \cong S_{2}$, либо $R_{2} \cong M_{2}(G F(2))$. Покажем, что второй случай невозможен. Если $R_{2} \cong M_{2}(G F(2))$, то множество $\left\{e_{11}, e_{21}, e_{11}+e_{21}\right\} \cup\left\{e_{22}, e_{21}+e_{22}, e_{1}\right\}$, где $e_{i j}-$ матричные единицы в $R_{2}$, образует граф $K_{3,3}$. Получаем противоречие. Следовательно, $R_{2} \cong S_{2}$.

Итак,

$$
e R e=G F(p) \dot{+} S_{2} \dot{+} e_{1} R e_{2} \dot{+} e_{2} R e_{1}, \quad S_{2} / J\left(S_{2}\right) \cong G F\left(p^{n}\right), \quad p=2,3 .
$$

Выясним, какие значения может принимать число $n$.

Пусть $p=3$. Предположим, что найдется ненулевой элемент $a$ из $e_{1} R e_{2}$. Тогда $\Gamma(R)$ содержит подграф, гомеоморфный $K_{5}$ : в качестве вершин графа $K_{5}$ возьмем элементы $e_{1}, 2 e_{1}, e_{2}, 2 e_{2}$ и $a$, причем вершины $e_{1}$ и $2 e_{1}$ соединим друг с другом с помощью дополнительной вершины $e_{2}+a$; аналогично, вершины $e_{2}$ и $2 e_{2}$ соединим с помощью вершины $e_{1}+a$. Получаем противоречие. Поэтому $e_{1} R e_{2}=(0)$. Аналогично доказывается, что $e_{2} R e_{1}=(0)$ и $e R(1-e)=(1-e) R e=(1-e) R(1-e)=(0)$. Поэтому $R=e R e=G F(3) \oplus S_{2}$, что противоречит неразложимости кольца $R$.

Итак, $p=2$ и $e R e=G F(2) \dot{+} S_{2} \dot{+} e_{1} R e_{2} \dot{+} e_{2} R e_{1}$, где $S_{2} / J\left(S_{2}\right) \cong G F\left(2^{n}\right)$. Покажем, что $S_{2} \cong G F(2)$. Предположим противное, а именно, пусть $\left|S_{2}\right| \geqslant 4$. Если существует ненулевой элемент $a \in e_{1} R e_{2}$, то множество $\left\{a_{1}, a_{2}, a_{3}\right\} \cup\left\{e_{1}, e_{1}+a, a\right\}$, где $a_{i} \in S_{2} \backslash\{0\}$, $i=1,2,3$, образует граф $K_{3,3}$; получаем противоречие. Следовательно, $e_{1} R e_{2}=(0)$. Аналогично $e_{2} R e_{1}=(0)$. Поэтому $e R e=G F(2) \oplus S_{2}$. Точно таким же образом легко показать, что $e R(1-e)=(1-e) R e=(1-e) R(1-e)=(0)$. Следовательно, $R=e R e=G F(2) \oplus S_{2}$, что противоречит неразложимости кольца $R$. Значит, $\left|S_{2}\right|=2$, то есть $S_{2} \cong G F(2)$.

Итак, мы доказали, что $R_{1} \cong G F(2)$ и $R_{2} \cong G F(2)$. Если $\left|e_{1} R e_{2}\right| \geqslant 4$, то $K_{3,3}$ содержится в $\Gamma(R)$. Действительно, для любых попарно различных ненулевых элементов $a_{k} \in e_{1} R e_{2}, k=1,2,3$, граф делителей нуля множества $\left\{a_{1}, a_{2}, a_{3}\right\} \cup\left\{e_{1}, e_{2}, e_{1}+a_{1}\right\}$ содержит в качестве подграфа $K_{3,3}$. Противоречие показывает, что $\left|e_{1} R e_{2}\right| \leqslant 2$. Аналогично и $\left|e_{2} R e_{1}\right| \leqslant 2$. Пусть $|e R(1-e)| \geqslant 4$ и $b_{1}, b_{2}, b_{3}$ - ненулевые попарно различные элементы из $e R(1-e)$. Тогда граф делителей нуля множества $\left\{b_{1}, b_{2}, b_{3}\right\} \cup\left\{e_{1}, e_{2}, e_{1}+e_{2}\right\}$ содержит $K_{3,3}$; получаем противоречие. Значит, $|e R(1-e)| \leqslant 2$. Аналогично получаем, что $|(1-e) R e| \leqslant 2$ и $|(1-e) R(1-e)| \leqslant 2$. Рассмотрим следующие подслучаи.

Подслучай А. $e R e=G F(2) \oplus G F(2)$. Предположим, что $(1-e) R(1-e) \neq(0)$. Если $e R(1-e) \neq(0)$, то для ненулевых элементов $a \in(1-e) R(1-e)$ и $b \in e R(1-e)$ граф делителей нуля множества $\left\{e_{1}, e_{2}, e_{1}+e_{2}\right\} \cup\{a, b, a+b\}$ содержит $K_{3,3}$. Полученное противоречие показывает, что $e R(1-e)=(0)$. Аналогично показывается, что и $(1-e) R e=(0)$. Поэтому кольцо $R$ является разложимым; получаем противоречие. Следовательно, $(1-e) R(1-e)=(0)$.

Пусть $e R(1-e) \neq(0)$ и $(1-e) R e \neq(0)$. По доказанному выше $|e R(1-e)|=2$ и $|(1-e) R e|=2$, то есть $2 R=(0)$. Итак, $R=\left\langle e_{1}\right\rangle \dot{+}\left\langle e_{2}\right\rangle \dot{+}\langle a\rangle \dot{+}\langle b\rangle$, где $a \in e R(1-e)$, $b \in(1-e) R e$. Так как $b a \in(1-e) R(1-e)=(0)$, справедливо равенство $b a=0$. Далее, $a b=e R e \cap J(R)=(0)$, то есть $a b=0$. Найдем $e_{i} a$ и $b e_{i}$. Поскольку $e_{i} a \in e R(1-e)$, элемент $e_{i} a$ равен 0 или $a$. Аналогично элемент $b e_{i}$ равен 0 или $b$. Если $e_{1} a=e_{2} a$, то $a=e a=\left(e_{1}+e_{2}\right) a=2 e_{1} a=0$; получаем противоречие. Таким образом, можно считать, что $e_{1} a=0$ и $e_{2} a=a$. Ясно также, что $b e_{1} \neq b e_{2}$. Если $b e_{1}=0$ 
и $b e_{2}=b$, то $\left\langle e_{1}\right\rangle$ отщепляется прямым слагаемым; получаем противоречие. Значит, $b e_{1}=b$ и $b e_{2}=0$. Но тогда $\Gamma(R)$ не планарен, поскольку граф делителей нуля множества $\left\{e_{1}, e_{1}+a, e_{2}\right\} \cup\{a, b, a+b\}$ содержит подграф $K_{3,3}$. Полученное противоречие доказывает, что случай, когда $e R(1-e) \neq(0)$ и $(1-e) R e \neq(0)$, невозможен.

Пусть $e R(1-e) \neq(0)$ и $(1-e) R e=(0)$, то есть $R=G F(2) \dot{+} G F(2) \dot{+} e R(1-e)$. Другими словами, $R=\left\langle e_{1}\right\rangle \dot{+}\left\langle e_{2}\right\rangle \dot{+}\langle a\rangle$, где $a-$ ненулевой элемент из $e R(1-e)$. Ясно, что $2 a=a^{2}=0, a e_{i}=0$. Так как $e_{i} a \in e R(1-e)$, элемент $e_{i} a$ равен 0 или $a$. Если $e_{1} a=0$, то $\left\langle e_{1}\right\rangle$ отщепляется прямым слагаемым, чего быть не должно. Следовательно, $e_{1} a=a$. Но тогда $e_{2} a=e_{2}\left(e_{1} a\right)=0$ и $\left\langle e_{2}\right\rangle$ отщепляется прямым слагаемым. Снова получили противоречие. Значит, случай, когда $e R(1-e) \neq(0)$ и $(1-e) R e=(0)$, невозможен. Случай, когда $e R(1-e)=(0)$ и $(1-e) R e \neq(0)$ рассматривается аналогично.

Подслучай В. $e R e=G F(2) \dot{+} G F(2) \dot{+} e_{1} R e_{2}$. Согласно доказанному выше, $\left|e_{1} R e_{2}\right|=2$. Возьмем ненулевой элемент $b \in e_{1} R e_{2}$. Ясно, что $b^{2}=0$. Если в $e R(1-e)$ существует ненулевой элемент $a$, то множество $\left\{e_{1}, b, e_{1}+b\right\} \cup\left\{a, a+b, e_{2}\right\}$ образует $K_{3,3}$; получаем противоречие. Следовательно, $e R(1-e)=(0)$. Для любого ненулевого элемента $a \in(1-e) R e$ граф делителей нуля множества $\left\{e_{2}, b, e_{2}+b\right\} \cup\left\{a, a+b, e_{1}\right\}$ содержит $K_{3,3}$; получаем противоречие. Значит, $(1-e) R e=(0)$. В силу прямой неразложимости кольца $R$ справедливы равенства $(1-e) R(1-e)=(0)$ и $R=e R e$. Итак, $R=\left\langle e_{1}\right\rangle \dot{+}\left\langle e_{2}\right\rangle \dot{+}\langle b\rangle$, где $2 e_{i}=2 b=0, b^{2}=0, b e_{1}=e_{2} b=0$. Если $e_{1} b=0$ или $b e_{2}=0$, то $\left\langle e_{1}\right\rangle$ или $\left\langle e_{2}\right\rangle$ соответственно отщепляется прямым слагаемым, чего быть не может. Следовательно, $e_{1} b=b e_{2}=b$ и $R \cong B_{2}$. Если $e R e=G F(2)+G F(2) \dot{+} e_{2} R e_{1}$, то аналогичным образом доказывается, что $R \cong B_{2}$.

Подслучай С. $e R e=G F(2) \dot{+} G F(2) \dot{+} e_{1} R e_{2} \dot{+} e_{2} R e_{1} . \quad$ Пусть $a_{12} \in e_{1} R e_{2}$ и $a_{21} \in e_{2} R e_{1}$ - ненулевые элементы. С одной стороны, $a_{i j} a_{j i} \in e_{i} R e_{i}=G F(2)$, а с другой стороны, $a_{i j} a_{j i} \in J(R)$. Поэтому $a_{12} a_{21}=0$ и $a_{21} a_{12}=0$. Тогда граф делителей нуля множества $\left\{a_{12}+e_{2}, e_{2}, a_{21}+e_{2}\right\} \cup\left\{a_{12}, a_{21}, e_{1}\right\}$ содержит в качестве подграфа $K_{3,3}$. Полученное противоречие показывает, что этот случай невозможен.

Итак, случай, когда $m=2$, полностью рассмотрен.

Случай 3. $m=1$. В этом случае

$$
R / J(R) \cong M_{n}(G F(q)), \quad e R e \cong M_{n}(S), \quad S / J(S) \cong G F(q) .
$$

Так же, как в случае, когда кольцо является полупростым, доказывается, что $e R e \cong S$ либо $e R e \cong M_{2}(G F(2))$. Пусть имеет место последний вариант: $e R e \cong M_{2}(G F(2))$. Если в $e R(1-e)$ существует хотя бы один ненулевой элемент $a$, то граф делителей нуля множества $\left\{e_{11}, e_{21}, e_{11}+e_{21}\right\} \cup\left\{e_{22}, e_{22}+e_{21}, a\right\}$, где $e_{i j}-$ матричные единицы в $e R e$, содержит $K_{3,3}$; получаем противоречие. Следовательно, $e R(1-e)=(0)$. Аналогично доказывается, что $(1-e) R e=(0)$ и $(1-e) R(1-e)=(0)$. Поэтому $J(R)=(0)$. Снова получили противоречие. Значит,

$$
e R e \cong S, \quad R / J(R) \cong G F(q) .
$$

Далее, граф $\Gamma(J(R))$ планарен и $J(R)$ нильпотентен, поэтому, в силу предложения 2.1 и леммы 2.4 из [6] и неразложимости кольца $R$, радикал Джекобсона $J(R)$ удовлетворяет одному из следующих условий:

(1) $J(R)^{2}=0,|J(R)| \leqslant 5$; 
(2) $J(R)^{3}=0,\left|J(R)^{2}\right|=2,|J(R)| \leqslant 8$;

(3) $J(R)^{3}=0,\left|J(R)^{2}\right|=3,|J(R)|=9$;

(4) $J(R)^{4}=0,\left|J(R)^{3}\right|=2,\left|J(R)^{2}\right|=4,|J(R)|=8$.

Обозначим через $N$ индекс нильпотентности $J(R)$. Тогда $J(R)^{N-1}$ является векторным пространством над полем $G F(q)$. Поэтому $|G F(q)| \leqslant\left|J(R)^{N-1}\right|$. Из всего сказанного выше можно сделать вывод, что кольцо $R$ удовлетворяет одному из следующих условий:

(1) $J(R)^{2}=0,|J(R)| \leqslant 5,|R| \leqslant 25$;

(2) $J(R)^{3}=0,\left|J(R)^{2}\right|=2,|J(R)| \leqslant 8,|R| \leqslant 16$;

(3) $J(R)^{3}=0,\left|J(R)^{2}\right|=3,|J(R)|=9,|R|=27$;

(4) $J(R)^{4}=0,\left|J(R)^{3}\right|=2,\left|J(R)^{2}\right|=4,|J(R)|=8,|R|=16$.

Предложение доказано.

Из доказательства предложения 1 вытекает следующее утверждение.

Следствие 1. Пусть $R$ - конечное неразложимое кольио с планарным графом делителей нуля, причем фактор-кольцо $R / J(R)$ является полем и $J(R) \neq(0)$. Тогда порядок кольца $R$ равен либо $p^{2}$ при $p=2,3,5$, либо $p^{3}$ при $p=2,3$, либо 16.

Заметим, что кольца порядков $p^{2}$ и $p^{3}$ полностью описаны (см., в частности, [9]). Кроме того, в работе [10] составлен полный список колец с единицей порядка $p^{4}$. Остается невыясненным вопрос о неразложимых кольцах без единицы порядка 16, для которых фактор-кольцо по радикалу Джекобсона является полем и граф делителей нуля планарен. Оказывается, что таких колец не существует. Доказательству этого факта посвящено следующее утверждение.

Предложение 2. Пусть $R$ - неразложимое кольияо без единицы порядка 16, причем фактор-кольцо $R / J(R)$ является полем. Тогда граф $\Gamma(R)$ не планарен.

Доказательство. Предположим, что существует такое неразложимое кольцо $R$ без единицы порядка 16 , что $\bar{R}=R / J(R)=G F\left(2^{n}\right), n \geqslant 1$, и граф $\Gamma(R)$ планарен. Пусть $e-$ идемпотент кольца $R$ такой, что его образ $\bar{e}$ в поле $\bar{R}$ является единицей. Согласно [9], справедливо соотношение

$$
e R e / J(e R e) \cong \bar{e} \bar{R} \bar{e} \cong \bar{R}
$$

то есть подкольцо $e R e$ является локальным кольцом. Далее в ходе доказательства мы будем использовать пирсовское разложение (1) кольца $R$. Поскольку в $R$ нет единицы, порядок подкольца $e R e$ не превосходит 8. Рассмотрим отдельные случаи в зависимости от того, чему равен порядок подкольца $e R e$.

Случай 1. Пусть $|e R e|=2$, то есть $e R e \cong G F(2)$. Предположим, что $|e R(1-e)| \geqslant 4$. Поскольку $(e R(1-e))^{2}=(0)$, для любых попарно различных ненулевых элементов $a, b, c \in e R(1-e)$ граф делителей нуля множества $\{a, b, c\} \cup\{e, e+a, e+b\}$ содержит в качестве подграфа $K_{3,3}$; получаем противоречие. Следовательно, $|e R(1-e)| \leqslant 2$. Аналогично доказывается, что и $|(1-e) R e| \leqslant 2$. 
Если $|(1-e) R(1-e)|=8$, то кольцо $R$ разложимо, и мы получаем противоречие. Предположим, что $|(1-e) R(1-e)|=4$. Тогда либо $|e R(1-e)|=2$, либо $|(1-e) R e|=2$. Если $|e R(1-e)|=2$, то для ненулевого элемента $a \in e R(1-e)$ и попарно различных ненулевых элементов $b, c, d \in(1-e) R(1-e)$ множество $\{b, c, d\} \cup\{e, a, e+a\}$ образует $K_{3,3}$; получаем противоречие. Следовательно, $e R(1-e)=(0)$ и $|(1-e) R e|=2$. Но и в этом случае точно таким же образом мы построим подграф $K_{3,3}$ и придем к противоречию. Отсюда заключаем, что $|(1-e) R(1-e)| \leqslant 2$. Следовательно, порядок каждой из четырех пирсовских компонент в разложении (1) равен 2.

Пусть $a \in e R(1-e), b \in(1-e) R e$ и $c \in(1-e) R(1-e)-$ ненулевые элементы. Тогда $2 R=0, a^{2}=b^{2}=0, c e=e c=c a=b c=0, a e=e b=0$. Поскольку $c^{2} \in(1-e) R(1-e)$, либо $c^{2}=0$ либо $c^{2}=c$. Однако $(1-e) R(1-e) \subseteq J(R)$, поэтому $c^{2}=0$. Далее, $a c \in e R(1-e)$. Если $a c=a$, то, умножив равенство справа на $c$, получим противоречие, а именно, $a=a c=a c^{2}=0$. Следовательно, $a c=0$. Точно так же доказывается, что $c b=0$. Тогда граф делителей нуля множества $\{e, e+c, c\} \cup\{a, b, a+c\}$ содержит $K_{3,3}$, то есть граф $\Gamma(R)$ не планарен. Получаем противоречие. Значит, этот случай невозможен.

Случай 2. Пусть $|e R e|=4$. Покажем сначала, что в этом случае $(1-e) R(1-e)=(0)$. Предположим противное, пусть $(1-e) R(1-e) \neq(0)$. Если, например, и $e R(1-e) \neq(0)$, то для ненулевых элементов $a \in e R(1-e), b \in(1-e) R(1-e)$ граф делителей нуля множества $\{a, b, a+b\} \cup\left\{a_{1}, a_{2}, a_{3}\right\}$, где $a_{i} \in e R e \backslash\{0\}, i=1,2,3$, содержит в качестве подграфа $K_{3,3}$; получаем противоречие. Следовательно, $e R(1-e)=(0)$. Точно так же и $(1-e) R e=(0)$. Значит, кольцо $R$ разложимо. Полученное противоречие доказывает, что $(1-e) R(1-e)=(0)$.

Далее, если $|e R(1-e)|=4$ или $|(1-e) R e|=4$, то граф $\Gamma(R)$ содержит $K_{3,3}$ : в качестве вершин подграфа $K_{3,3}$ берем три ненулевых элемента из $e R e$ и три ненулевых элемента из $e R(1-e)$ или $(1-e) R e$ соответственно. Получаем противоречие. Поэтому $|e R(1-e)|=|(1-e) R e|=2$. Подкольцо $e R e-$ локальное кольцо порядка 4, поэтому $e R e$ изоморфно одному из следующих колец: $G F(4), \mathbf{Z}_{4}, \mathbf{Z}_{2}[x] /\left(x^{2}\right)$ (см. с.54 в [9]). Рассмотрим каждый из этих случаев отдельно.

Пусть $e R e=G F(4)$. Поскольку $e R(1-e)$ является правым модулем над полем $e R e$, справедливо соотношение $|e R e| \leqslant|e R(1-e)|=2$. Получаем противоречие. Значит, этот случай невозможен.

Пусть $e R e=\mathbf{Z}_{4}$ и $a \in e R(1-e), b \in(1-e) R e-$ ненулевые элементы. Ясно, что $2 e \neq 0,4 e=2 a=2 b=0, a^{2}=b^{2}=0$, $a e=e b=0$. Кроме того, справедливо включение $b a \in(1-e) R(1-e)=(0)$, то есть $b a=0$. Элементы $a, b, 2 e, 2 e+a, 2 e+b$ образуют $K_{5}$; получаем противоречие. Значит, и этот случай невозможен.

Пусть, наконец, $e R e=\mathbf{Z}_{2}[x] /\left(x^{2}\right)$. Тогда $2 R=(0)$ и $R=\langle e\rangle \dot{+}\langle t\rangle \dot{+}\langle a\rangle \dot{+}\langle b\rangle$, где $e t=t e=t, t^{2}=0, a \in e R(1-e), b \in(1-e) R e$. Ясно, что $a t=t b=0, a^{2}=0, b^{2}=0$ и $b a=0$. Далее, $t a \in e R(1-e)=\{0, a\}$. Если $t a=a$, то, домножив это равенство слева на $t$, приходим к равенствам $0=t^{2} a=t a=a$; получаем противоречие. Значит, $t a=0$. Аналогично и $b t=0$. Поэтому элементы $a, b, t, a+t, b+t$ образуют $K_{5}$. Получаем противоречие. Значит, случай, когда $|e R e|=4$, невозможен.

Случай 3. Пусть $|e R e|=8$. Поскольку кольцо $R$ неразложимо, выполняется одно из следующих условий: либо $R=e R e \dot{+} e R(1-e),|e R(1-e)|=2$, либо $R=e R e \dot{+}(1-e) R e$, $|(1-e) R e|=2$. Будем в дальнейшем считать, что $R=e R e+e R(1-e)$ (случай, когда выполняется равенство $R=e R e+(1-e) R e$, рассматривается аналогично). Пусть 
$d-$ ненулевой элемент из $e R(1-e)$, то есть $e R(1-e)=\{0, d\}$. Ясно, что $e d=d$ и $2 d=d^{2}=d e=0$. Поскольку $e R e-$ локальное кольцо порядка 8, согласно [9] $e R e$ изоморфно одному из колец $G F(8), \mathbf{Z}_{8}, \mathbf{Z}_{2}[x, y] /(x, y)^{2}, Z_{2}[x] /\left(x^{3}\right), \mathbf{Z}_{4}[x] /\left(2 x, x^{2}-2 \gamma\right)$, $\gamma=0,1$.

Точно таким же образом, как это было сделано в случае 2 , получаем, что $e R e$ не может быть изоморфно полю $G F(8)$. Рассмотрим отдельно каждый из оставшихся вариантов.

Если $e R e=\mathbf{Z}_{8}$, то $8 e=0$ и $4 e \neq 0$. В этом случае граф делителей нуля множества $\{2 e, 2 e+d, 6 e\} \cup\{d, 4 e, 4 e+d\}$ содержит в качестве подграфа $K_{3,3}$; получаем противоречие.

Пусть $e R e=\mathbf{Z}_{2}[x, y] /(x, y)^{2}$. Ясно, что $d \bar{x}=d \bar{y}=0$. Далее, произведение $\bar{x} d$ равно 0 или $d$. Если $\bar{x} d=d$, то, домножив слева на $\bar{x}$, получим, что $0=\bar{x} d=d$; получаем противоречие. Значит, $\bar{x} d=0$. Аналогично и $\bar{y} d=0$. Тогда элементы $d, \bar{x}, \bar{y}, \bar{x}+d$, $\bar{y}+d$ образуют граф $K_{5}$; получаем противоречие.

Пусть теперь $e R e=\mathbf{Z}_{2}[x] /\left(x^{3}\right)$. Ясно, что $d \bar{x}=0$. Поскольку $\bar{x} d \in e R(1-e)$ и $\bar{x} d$ не может быть равно $d$, справедливо равенство $\bar{x} d=0$. В этом случае элементы $\left\{\bar{x}^{2}, d, \bar{x}^{2}+d\right\} \cup\left\{\bar{x}, \bar{x}+d, \bar{x}+\bar{x}^{2}\right\}$ образуют $K_{3,3}$; получаем противоречие.

Остался вариант $e R e=\mathbf{Z}_{4}[x] /\left(2 x, x^{2}-2 \gamma\right)$, где $\gamma=0,1$. Так как $d \bar{x}=2 \bar{x}=4 e=0$ и $2 e \neq 0$, граф делителей нуля множества $\{\bar{x}, \bar{x}+d, \bar{x}+2 e\} \cup\{d, d+2 e, 2 e\}$ содержит $K_{3,3}$; получаем противоречие.

Итак, мы рассмотрели все возможные варианты и показали, что граф делителей нуля любого неразложимого кольца без единицы порядка 16, для которого фактор-кольцо по радикалу Джекобсона является полем, непланарен.

Теперь мы можем составить полный список конечных неразложимых ненильпотентых колец, имеющих планарные графы делителей нуля.

Теорема 1. Пусть $R$ - конечное неразложимое ненильпотентное кольчо. Тогда граф $\Gamma(R)$ планарен в том и только в том случае, когда $R$ изоморфно одному из следующих колеи:

(1) $G F\left(p^{n}\right)$;

(2) $M_{2}(G F(2))$;

(3) $B_{2}$;

(4) $\mathbf{Z}_{p^{2}}$, где $p=2,3,5$;

(5) $G F(p)[x] /\left(x^{2}\right)$, где $p=2,3,5$;

(6) $G F(2)[x, y] /(x, y)^{2}$;

(7) $G F(p)[x] /\left(x^{3}\right)$, где $p=2,3$;

(8) $\mathbf{Z}_{p^{2}}[x] /\left(p x, x^{2}-\gamma p\right)$, где $p \leqslant 3$ и $\gamma=0,1$ при $p=2, \gamma= \pm 1$ при $p=3$;

(9) $\mathbf{Z}_{p^{3}}$, где $p=2,3$;

(10) $G F(4)[x] /\left(x^{2}\right)$;

(11) $G F(2)[x] /\left(x^{4}\right)$;

(12) $G F(2)[x, y] /\left(x^{2}-y^{2}, x y\right)$; 
(13) $G F(2)[x, y] /\left(x^{2}, y^{2}\right)$;

(14) $\mathbf{Z}_{4}[x] /\left(x^{2}+x+1\right)$;

(15) $\mathbf{Z}_{4}[x] /\left(2 x, x^{3}-2\right)$;

(16) $\mathbf{Z}_{4}[x] /\left(x^{2}-2\right)$;

(17) $\mathbf{Z}_{4}[x] /\left(x^{2}-2 x-2\right)$;

(18) $\mathbf{Z}_{4}[x, y] /\left(x^{2}-2, x y, y^{2}-2,2 x\right)$;

(19) $\mathbf{Z}_{4}[x, y] /\left(x^{2}, x y-2, y^{2}\right)$;

(20) $\mathbf{Z}_{4}[x] /\left(x^{2}\right)$;

(21) $\mathbf{Z}_{4}[x] /\left(x^{2}-2 x\right)$;

(22) $\mathbf{Z}_{8}[x] /\left(2 x, x^{2}-4\right)$;

(23) $\mathbf{Z}_{16}$;

(24) $A_{p}, A_{p}^{0}$, где $p=2,3$;

(25) $\langle f\rangle \dot{+}\langle a\rangle$, где $4 f=2 a=0, f^{2}=f, f a=a$, af $=0, a^{2}=0$;

(26) $\langle f\rangle \dot{+}\langle a\rangle$, где $4 f=2 a=0, f^{2}=f, f a=0, a f=f, a^{2}=0$;

(27) $\langle f\rangle \dot{+}\langle a\rangle \dot{+}\langle b\rangle$, где $2 f=2 a=2 b=0, f^{2}=f$, af $=f a=a, b f=a$, $a^{2}=b^{2}=f b=a b=b a=0 ;$

(28) $\langle f\rangle \dot{+}\langle a\rangle \dot{+}\langle b\rangle$, где $2 f=2 a=2 b=0, f^{2}=f, f b=b$, af $=f a=a$, $a^{2}=b^{2}=b f=a b=b a=0$;

(29) $\langle f\rangle \dot{+}\langle a\rangle \dot{+}\langle b\rangle$, где $2 f=2 a=2 b=0, f^{2}=f, f b=b$, af $=a, a^{2}=b^{2}=a b=$ $b a=b f=f a=0$;

(30) $C_{4}$

Доказательство. Пусть $R$ - конечное неразложимое ненильпотентное кольцо с планарным графом $\Gamma(R)$. По предложению 1 , либо $R$ изоморфно одному из колец (1)-(3) из условия теоремы, либо $R / J(R)$ является полем, причем $J(R) \neq(0)$. Из рис. 1 видно, что кольца (2) и (3) имеют планарные графы делителей нуля. Поэтому мы можем считать, что $R / J(R)$ является полем и $J(R) \neq(0)$. По следствию 1 , порядок $R$ может быть равен либо $p^{2}$ при $p=2,3,5$, либо $p^{3}$ при $p=2,3$, либо 16. Как отмечалось выше, конечные кольца порядков $p^{2}$ и $p^{3}$ полностью описаны [9]. Кроме того, известен полный список конечных колец с единицей порядка $p^{4}[10]$. По предложению 2, не существует неразложимых колец без единицы порядка 16, для которых фактор-кольцо по радикалу Джекобсона является полем, а граф делителей нуля планарен. Далее, коммутативные неразложимые кольца с единицей, имеющие планарные графы делителей нуля, были описаны в работе [5]. Это кольца (4)-(23) из формулировки теоремы и конечные поля (1).

Таким образом, для завершения доказательства осталось пройтись по списку конечных неразложимых колец порядков $p^{2}, p^{3}$ и локальных колец порядка 16 и выяснить, какие из 
них имеют планарные графы делителей нуля, при этом необходимо учитывать, что факторкольцо по радикалу Джекобсона является полем, и опускать коммутативные кольца с единицей.

Пусть $|R|=p^{2}, p=2,3,5$. Тогда, согласно [9], $R$ может быть изоморфно $A_{p}$ либо $A_{p}^{0}$. Очевидно, что $\Gamma\left(A_{2}\right)$ и $\Gamma\left(A_{2}^{0}\right)$ планарны. Граф $\Gamma\left(A_{3}\right)$ получается из графа $K_{2,6}$ с долями $\left\{e_{12}, 2 e_{12}\right\}$ и $\left\{\alpha e_{11}+\beta e_{12} ; \alpha=1,2, \beta=0,1,2\right\}$, если соединить ребром вершины $e_{12}$ и $2 e_{12}$. Поэтому граф $Г\left(A_{3}\right)$ планарен. Кольцо $A_{3}^{0}$ антиизоморфно кольцу $A_{3}$, поэтому $\Gamma\left(A_{3}^{0}\right)=\Gamma\left(A_{3}\right)$. Далее, $\Gamma\left(A_{5}\right)$ не планарен, поскольку множество $\left\{e_{11}, 2 e_{11}, 3 e_{11}\right\} \cup\left\{e_{12}, 2 e_{12}, 3 e_{12}\right\}$ образует $K_{3,3}$. Следовательно, не планарен и $\Gamma\left(A_{5}^{0}\right)$.

Рассмотрим теперь случай, когда $|R|=p^{3}, p=2,3$. Тогда, согласно [9], $R$ удовлетворяет одному из следующих условий:

(А) $R=\langle f\rangle \dot{+}\langle a\rangle$, где $p^{2} f=p a=0$ и выполняется одно из следующих соотношений:

(A-1) $f^{2}=f, f a=a, a f=0, a^{2}=0$;

(A-2) $f^{2}=f, f a=0, a f=a, a^{2}=0 ;$

(В) $R=\langle f\rangle \dot{+}\langle a\rangle \dot{+}\langle b t\rangle$, причем $p f=p a=p b=0$ и умножение задается одним из следующих соотношений:

(B-1) $f^{2}=f, a f=a, b f=b, a^{2}=b^{2}=f a=f b=a b=b a=0$;

(B-2) $f^{2}=f$, $a f=f a=a, b f=a, a^{2}=b^{2}=f b=a b=b a=0$;

(B-3) $f^{2}=f, f b=b, a f=f a=a, a^{2}=b^{2}=b f=a b=b a=0$;

(B-4) $f^{2}=f, f b=b, a f=a, a^{2}=b^{2}=a b=b a=b f=f a=0$;

(B-5) $f^{2}=f, f a=a, f b=b, a^{2}=b^{2}=a f=b f=a b=b a=0$.

Проверим, какие из этих колец имеют планарные графы делителей нуля. Графы делителей нуля колец В-1 и В-5 не планарны, поскольку в обоих случаях элементы $\{f, f+a, f+b\} \cup\{a, a+b, b\}$ образуют $K_{3,3}$.

В кольце А-1 при $p=2$ вершины $a, 2 f, 2 f+a$ образуют треугольник, а остальные вершины $f, f+a, 3 f, 3 f+a$ смежны только с вершиной $a$, то есть являются вершинами степени 1. Точно такой же вид при $p=2$ имеет граф делителей нуля кольца А-2, антиизоморфного кольцу А-1. Таким образом, графы делителей нуля колец А-1 и А-2 при $p=2$ планарны. В кольцах А-1 и А-2 при $p=3$ графы делителей нуля элементов $\{6 f, 6 f+a, 6 f+2 a\} \cup\{a, 2 a, 3 f\}$ содержат $K_{3,3}$.

Далее, в кольцах В-2, В-3 и В-4 при $p=2$ элементы $a, b, a+b$ образуют треугольник. Остается выяснить степени смежности вершин $f, f+a, f+b, f+a+b$. В графе делителей нуля кольца В-2 при $p=2$ все эти вершины смежны лишь с $b$ и $a+b$, то есть являются вершинами степени 2. В графе делителей нуля кольца В-3 при $p=2$ вершины $f, f+a, f+b, f+a+b$ имеют степень 1 , поскольку все они смежны лишь с $b$. Наконец, в графе делителей нуля кольца В-4 при $p=2$ эти вершины смежны лишь с $a$ и $b$, то есть являются вершинами степени 2. Таким образом, графы делителей нуля колец В-2, B-3 и B-4 при $p=2$ планарны (в условии теоремы это кольца (27)-(29)). Кольца В-2, В-3 и B-4 при $p=3$ имеют непланарные графы делителей нуля, так как во всех этих случаях элементы $a, 2 a, b, 2 b, a+b$ образуют подграф $K_{5}$.

Теперь необходимо из списка локальных некоммутативных колец порядка 16 выбрать те, что имеют планарные графы делителей нуля. Этим самым доказательство теоремы будет завершено.

Итак, пусть $R$ - некоммутативное локальное кольцо порядка 16. Согласно [10], кольцо $R$ изоморфно одному из следующих колец: 
(C-1) $C_{4}$;

(C-2) $\mathbf{Z}_{4}\langle x, y\rangle /\left(x^{2}-2, y^{2}-2 \varphi, 2 x, 2 y, x y-2, y x\right), \varphi=0,1$;

(C-3) $J^{\#}$, где $J-$ нильпотентная $\mathbf{Z}_{2}$-алгебра с $\mathbf{Z}_{2}$-базисом $\{a, b, c\}$ таким, что $c^{2}=a c=$ $c a=b c=c b=0, a^{2}=a b=c, b a=0, b^{2}=\gamma c, \gamma \in \mathbf{Z}_{2}$.

Для локального конечного кольца множество делителей нуля кольца совпадает с радикалом Джекобсона этого кольца, поэтому $\Gamma(R)=\Gamma(J(R))$. Порядок радикала Джекобсона кольца C-1 равен 4, поэтому граф делителей нуля этого кольца планарен.

Кольца С-2 при любом значении $\varphi$ имеют непланарные графы делителей нуля. Действительно, если $\varphi=0$, то вершины $\bar{x}, \bar{y}, \overline{2}, \bar{x}+\bar{y}, \bar{y}+\overline{2}$ образуют $K_{5}$. При $\varphi=1$ граф делителей нуля множества $\{\bar{x}, \overline{2}, \bar{x}+\overline{2}\} \cup\{\bar{y}, \bar{y}+\overline{2}, \bar{x}+\bar{y}+\overline{2}\}$ содержит $K_{3,3}$.

Осталось рассмотреть случай, когда $R$ изоморфно одному из колец С-3. Так как $J(R)=J$, справедливо равенство $\Gamma(R)=\Gamma(J)$. Согласно [6] графы делителей нуля колец C-3 являются непланарными при любом значении $\gamma$.

Теорема доказана.

Прежде чем приступить к описанию конечных разложимых ненильпотентных колец с планарными графами делителей нуля, докажем две несложные леммы, которые понадобятся нам в дальнейшем.

Лемма 1. Пусть $R$ - неразложимое конечное кольцьо. Тогда $\Gamma(R)=K_{1}$ в том и только в том случае, когда $R$ изоморфно одному из колец $N_{0,2}, \mathbf{Z}_{4}, \mathbf{Z}_{2}[x] /\left(x^{2}\right)$.

Доказательство. Пусть $R-$ неразложимое конечное кольцо и $\Gamma(R)=K_{1}$. Если $R=J(R)$, то $|R|=2$, то есть $R \cong N_{0,2}$ (см. с. 53 в [9]).

Пусть $R \neq J(R)$. В силу предложения 1 справедливо равенство $R / J(R)=G F(q)$, причем $J(R) \neq(0)$. Поскольку $J(R) \neq(0)$ и $\Gamma(R)=K_{1}$, справедливо равенство $|J(R)|=2$. Поэтому $J(R)$ является векторным пространством над полем $R / J(R)$, то есть $|R / J(R)| \leqslant|J(R) t|$. Отсюда следует, что $|R|=4$. Согласно [9], кольцо $R$ изоморфно одному из колец $\mathbf{Z}_{4}, \mathbf{Z}_{2}[x] /\left(x^{2}\right), A_{2}, A_{2}^{0}$. Заметив, что $\Gamma\left(\mathbf{Z}_{4}\right)=\Gamma\left(\mathbf{Z}_{2}[x] /\left(x^{2}\right)\right)=K_{1}$ и $\Gamma\left(A_{2}\right)=\Gamma\left(A_{2}^{0}\right)=K_{1,2}$, мы тем самым завершим доказательство леммы.

Лемма 2. Пусть $R$ - неразложимое конечное кольцьо. Тогда $\Gamma(R)=K_{2}$ в том и только в том случае, когда $R$ изоморфно одному из колец $N_{0,3}, \mathbf{Z}_{9}, \mathbf{Z}_{3}[x] /\left(x^{2}\right)$.

Доказательство. Пусть $R-$ неразложимое конечное кольцо и $\Gamma(R)=K_{2}$. Если $R=J(R)$, то $|R|=3$ и $R \cong N_{0,3}$ (см. с. 53 в [9]).

Рассмотрим теперь случай, когда $R \neq J(R)$. Из предложения 1 следует, что $R / J(R)=G F(q)$ и $J(R) \neq(0)$. Так как $J(R) \neq(0)$ и $\Gamma(R)=K_{2}$, справедливо неравенство $|J(R)| \leqslant 3$. Точно так же, как это было сделано в доказательстве предыдущей леммы, мы получаем, что порядок кольца $R$ равен 4 или 9. Из доказательства леммы 1 видно, что не существует ненильпотентных неразложимых колец порядка 4 с графом делителей нуля, равным $K_{2}$. Пусть $|R|=9$. Согласно [9], кольцо $R$ изоморфно одному из колец $\mathbf{Z}_{9}, \mathbf{Z}_{3}[x] /\left(x^{2}\right), A_{3}, A_{3}^{0}$. Легко проверить, что лишь два из этих колец имеют граф делителей нуля, равный $K_{2}$, а именно, $\mathbf{Z}_{9}$ и $\mathbf{Z}_{3}[x] /\left(x^{2}\right)$.

Теперь мы можем приступить к доказательству следующей теоремы, которая завершает описание конечных ненильпотентных колец с планарными графами делителей нуля.

Теорема 2. Пусть $R-$ конечное разложимое ненильпотентное кольцио. Тогда граф $\Gamma(R)$ планарен в том и только в том случае, когда $R$ изоморфно одному из следующих колец: 
(1) $G F(2) \oplus G F(2) \oplus G F(p), p=2,3$;

(2) $G F(2) \oplus \mathbf{Z}_{8}$;

(3) $G F(2) \oplus \mathbf{Z}_{2}[x] /\left(x^{3}\right)$;

(4) $G F(2) \oplus \mathbf{Z}_{4}[x] /\left(2 x, x^{2}-2\right)$;

(5) $G F(p) \oplus \mathbf{Z}_{q^{2}}, p=2,3, q=2,3$;

(6) $G F(p) \oplus \mathbf{Z}_{q}[x] /\left(x^{2}\right), p=2,3, q=2,3$;

(7) $G F(p) \oplus G F\left(q^{n}\right)$, где $p=2,3, n \geqslant 1$ и $q$ - простое число;

(8) $N_{0,2} \oplus G F(2) \oplus G F(2)$;

(9) $N_{0, p} \oplus G F\left(q^{n}\right)$, где $q-$ простое число, $n \geqslant 1$ и $p=2,3$;

(10) $N_{0,2} \oplus \mathbf{Z}_{4}$;

(11) $N_{0,2} \oplus \mathbf{Z}_{2}[x] /\left(x^{2}\right)$;

(12) $G F(2) \oplus N_{4}$;

(13) $G F(2) \oplus N_{2,2}$;

(14) $G F(2) \oplus A_{2}, G F(2) \oplus A_{2}^{0}$.

Доказательство. Коммутативные разложимые кольца с единицей, имеющие планарные графы делителей нуля, описаны в [4]. Это кольца (1)-(7) из условия теоремы. Поэтому в дальнейшем мы будем полагать, что $R-$ конечное разложимое ненильпотентное кольцо с планарным графом делителей нуля, причем либо $R$ не является коммутативным, либо в $R$ нет единицы.

Пусть $R=R_{1} \oplus R_{2} \oplus \ldots \oplus R_{m}$, где $m>1$ и $R_{i}$ - неразложимые кольца, $i=1, \ldots, m$.

Заметим, что если $m \geqslant 4$, то $K_{3,3}$ содержится в $\Gamma(R)$. Действительно, для любых ненулевых элементов $r_{k} \in R_{k}, k=1,2,3,4$, граф делителей нуля множества $\left\{r_{1}, r_{1}+r_{2}, r_{2}\right\} \cup\left\{r_{3}, r_{3}+r_{4}, r_{4}\right\}$ содержит $K_{3,3}$, получаем противоречие. СледовательHO, $m \leqslant 3$.

Случай 1. $m=3$. Предположим, что какие-то два из трех колец $R_{i}, i=1,2,3$, имеют порядки, превосходящие 2 , например, $\left|R_{1}\right| \geqslant 3$ и $\left|R_{2}\right| \geqslant 3$. Тогда для любых элементов $r_{i}, s_{i} \in R_{i}, r_{i} \neq s_{i}, i=1,2$, и любого ненулевого элемента $r \in R_{3}$, граф $\Gamma(R)$ содержит подграф, гомеоморфный $K_{5}$ : в качестве вершин графа $K_{5}$ возьмем элементы $r_{1}, s_{1}, r, r_{2}$, $s_{2}$, причем вершины $r_{1}$ и $s_{1}$ соединим друг с другом с помощью дополнительной вершины $r_{2}+r$, а вершины $r_{2}$ и $s_{2}$ соединим с помощью вершины $r_{1}+r$. Мы получили противоречие. Значит, по крайней мере порядки двух колец $R_{i}$ равны 2 . Не нарушая общности, мы можем полагать, что, например, $\left|R_{1}\right|=\left|R_{2}\right|=2$. Следовательно, $R_{1}, R_{2} \cong G F(2), N_{0,2}$ (см. с. 51 в [9]). Если $\left|R_{3}\right| \geqslant 4$, то множество $\left\{r_{1}, r_{1}+r_{2}, r_{2}\right\} \cup\{a, b, c$,$\} , где r_{i} \in R_{i} \backslash\{0\}$, $i=1,2$, и $a, b, c$, - попарно различные ненулевые элементы из $R_{3}$, образует $K_{3,3}$; получаем противоречие. Значит, $\left|R_{3}\right| \leqslant 3$, то есть $R_{3} \cong G F(p), N_{0, p}$, где $p=2$, 3. Таким образом, кольцо $R$ коммутативно, поэтому мы можем считать, что в $R$ нет единицы.

Пусть $R_{3} \cong N_{0,3}$. Поскольку $\Gamma\left(N_{0,2} \oplus N_{0,3}\right)=K_{5}$, получаем, что $R_{1}, R_{2} \cong G F(2)$ в силу планарности графа $\Gamma(R)$. Граф $\Gamma\left(G F(2) \oplus G F(2) \oplus N_{0,3}\right)$ не является планарным, 


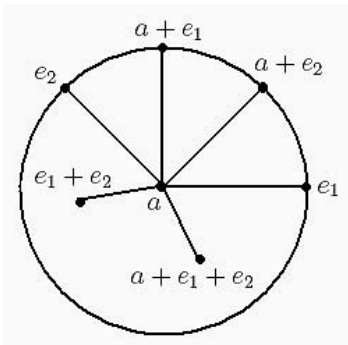

Pис. 2. Граф $\Gamma\left(N_{0,2} \oplus G F(2) \oplus G F(2)\right)$

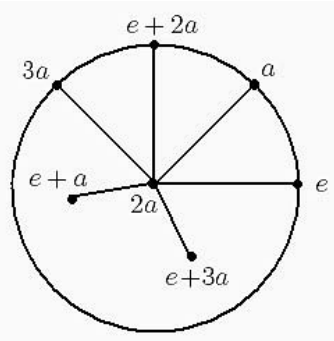

Рис. 3. Граф $\Gamma\left(G F(2) \oplus N_{4}\right)$

так как множество $\left\{e_{1}, e_{1}+a, e_{1}+b\right\} \cup\left\{e_{2}, e_{2}+a, e_{2}+b\right\}$, где $e_{i}-$ единица в $R_{i}$, $i=1,2$, и $a, b \in N_{0,3}$, образует $K_{3,3}$; получаем противоречие. Следовательно, $R_{3}$ не может быть изоморфно $N_{0,3}$. Поскольку $R$ не содержит единицы и не является нильпотентным, в разложении кольца $R$ содержится либо одна, либо две нильпотентные компоненты, причем эти нильпотентные компоненты, как только что было показано, изоморфны $N_{0,2}$.

Пусть в разложении кольца $R$ имеется только одна нильпотентная компонента. Тогда $R=N_{0,2} \oplus G F(2) \oplus G F(p)$, где $p=2,3$. Пусть, далее, $e_{1}-$ единица в $G F(2), e_{2}-$ единица в $G F(p)$ и $a-$ ненулевой элемент из $N_{0,2}$. Если $p=3$, то граф делителей нуля множества $\left\{e_{1}, e_{1}+a, a\right\} \cup\left\{e_{2}, e_{2}+a, 2 e_{2}\right\}$ образует $K_{3,3}$; получаем противоречие. Следовательно, $p=2$ и $R=N_{0,2} \oplus G F(2) \oplus G F(2)$. Как видно из рис. 2, граф $\Gamma\left(N_{0,2} \oplus G F(2) \oplus G F(2)\right)$ планарен.

Пусть в разложении $R$ есть 2 нильпотентные компоненты, т.е. $R=N_{0,2} \oplus N_{0,2} \oplus G F(p)$, где $p=2,3$. Если $a, b$ - два различных ненулевых элемента из $N_{0,2} \oplus N_{0,2}$, а $e-$ единица в $G F(p)$, то граф делителей нуля множества $\{a, b, a+b\} \cup\{e, e+a, e+b\}$ содержит в качестве подграфа $K_{3,3}$. Значит, $\Gamma\left(N_{0,2} \oplus N_{0,2} \oplus G F(p)\right)$ не планарен.

Таким образом, случай, когда $m=3$, полностью рассмотрен.

Случай 2. $m=2$. Если порядки колец $R_{1}$ и $R_{2}$ больше 3 , то $K_{3,3} \subseteq \Gamma(R)$, чего быть не может. Поэтому, не нарушая общности, можем полагать, что $\left|R_{1}\right| \leqslant 3$. Следовательно, $R_{1}$ изоморфно $G F(p)$ или $N_{0, p}$, где $p=2,3$. Рассмотрим каждый из этих вариантов отдельно.

Подслучай А. $R_{1} \cong N_{0,3}$. Пусть $0 \neq a \in R_{1}$. Предположим, что в $R_{2}$ найдутся два различных ненулевых элемента $\beta, \gamma$, такие, что $\gamma \beta=0$. Тогда элементы 
$\{\gamma+a, \gamma+2 a, \gamma\} \cup\{a, 2 a, \beta\}$ образуют $K_{3,3}$; получаем противоречие. В силу связности графа делителей нуля (см. [11]), либо в $R_{2}$ нет делителей нуля, то есть $R_{2}$ является конечным полем, либо $\Gamma\left(R_{2}\right)=K_{1}$. Пусть $\Gamma\left(R_{2}\right)=K_{1}$. Тогда в $R_{2}$ найдется такой ненулевой элемент $\lambda$, что $\lambda^{2}=0$. Однако и в этом случае граф $\Gamma(R)$ не является планарным, поскольку элементы $a, 2 a, \lambda, \lambda+a, \lambda+2 a$ образуют подграф $K_{5}$. Противоречие свидетельствует о том, что $R_{2}=G F(q)$, где $q=p^{n}, p-$ простое число и $n>0$. Заметим, что граф $\Gamma\left(N_{0,3} \oplus G F(q)\right)$ планарен. Действительно, если $G F(q)=\left\{0, \xi_{1}, \xi_{2}, \ldots, \xi_{q-1},\right\}$ то граф $\Gamma\left(N_{0,3} \oplus G F(q)\right)$ получается из полного двудольного графа с долями $\{a, 2 a\}$ и $\left\{\xi_{i}, a+\xi_{i}, 2 a+\xi_{i} ; i=1,2, \ldots, q-1\right\}$ путем добавления ребра между вершинами $a$ и $2 a$.

Подслучай В. $R_{1} \cong N_{0,2}$. Предположим, что в $R_{2}$ существует три попарно различных ненулевых делителя нуля $\beta, \delta, \gamma$ такие, что, например, вершина $\beta$ смежна с $\delta$ и $\gamma$. Возьмем $a \in R_{1}, a \neq 0$. Тогда элементы $\{\beta, \beta+a, a\} \cup\{\delta, \gamma, \gamma+a\}$ образуют подграф $K_{3,3}$; получаем противоречие. Значит, либо $R_{2}$ не имеет делителей нуля, либо $\Gamma\left(R_{2}\right)=K_{1}$, либо $\Gamma\left(R_{2}\right)=K_{2}$. В силу лемм $1-2$ и того условия, что кольцо $R$ не является нильпотентным, $R_{2}$ изоморфно одному из колец $G F(q), \mathbf{Z}_{4}, \mathbf{Z}_{2}[x] /\left(x^{2}\right), \mathbf{Z}_{9}, \mathbf{Z}_{3}[x] /\left(x^{2}\right)$. Заметим, что граф $\Gamma\left(N_{0,2} \oplus G F(q)\right)$ является звездой, поэтому планарен. Граф $\Gamma\left(N_{0,2} \oplus \mathbf{Z}_{4}\right)$ получается из графа $K_{1,6}$ с долями $\{a\}$ и $\{\overline{1}, \overline{2}, \overline{3}, a+\overline{1}, a+\overline{2}, a+\overline{3}\}$, если соединить ребром вершины $\overline{2}$ и $a+\overline{2}$. Поэтому и этот граф планарен. Далее, $\Gamma\left(N_{0,2} \oplus \mathbf{Z}_{2}[x] /\left(x^{2}\right)\right)=\Gamma\left(N_{0,2} \oplus \mathbf{Z}_{4}\right)$. Граф $\Gamma\left(N_{0,2} \oplus \mathbf{Z}_{9}\right)$ не планарен, так как элементы $\overline{3}, \overline{6}, a, a+\overline{3}, a+\overline{6}$ образуют $K_{5}$. Граф $\Gamma\left(N_{0,2} \oplus \mathbf{Z}_{3}[x] /\left(x^{2}\right)\right)$ также не планарен, так как элементы $\bar{x}, 2 \bar{x}, a, a+\bar{x}, a+2 \bar{x}$ образуют $K_{5}$.

Подслучай С. $R_{1} \cong G F(p), p=2,3$. Пусть $e-$ единица в $R_{1}$. Предположим, что в $\Gamma\left(R_{2}\right)$ существует по крайней мере четыре вершины. Пусть одна из вершин (обозначим ее через $a$ ) имеет степень, большую 2, и $b, c, d$ - вершины, смежные с $a$. Тогда элементы $\{e, a, e+a\} \cup\{b, c, d\}$ образуют $K_{3,3}$. Получаем противоречие. Следовательно, в $\Gamma\left(R_{2}\right)$ степени всех вершин равны 1 или 2 . Поэтому $\Gamma\left(R_{2}\right)$ является либо цепью длины, большей или равной трем, либо циклом длины, большей или равной четырем. Заметим, что $R_{2}$ не может быть локальным или нильпотентным кольцом, поскольку в графах делителей нуля локальных и нильпотентных колец существует вершина, смежная со всеми остальными вершинами. Поэтому из доказательства теоремы 1 следует, что колец с такими графами делителей нуля не существует. Значит, в $\Gamma\left(R_{2}\right)$ содержится не более трех вершин.

Предположим, что в $\Gamma\left(R_{2}\right)$ существует не более двух вершин. Тогда по леммам 1 и $2 R_{2}$ изоморфно одному из колец $N_{0, p}, \mathbf{Z}_{p^{2}}, \mathbf{Z}_{p}[x] /\left(x^{2}\right), p=2,3$. Нами уже описаны случаи, когда одна из двух компонент является нильпотентным кольцом (подслучай $\mathrm{A}$ и подслучай $\mathrm{B})$, поэтому мы можем считать, что $R_{2}$ не является нильпотентным. Тогда $R_{2}$ (а следовательно, и $R$ ) является коммутативным кольцом с единицей. Однако, конечные коммутативные кольца с единицей, имеющие планарные графы делителей нуля, полностью описаны.

Итак, осталось рассмотреть случай, когда в $R_{2}$ существует точно три ненулевых делителя нуля. В силу связности графа делителей нуля, $\Gamma\left(R_{2}\right)$ равен $K_{1,2}$, либо $K_{3}$. Пусть $D$ - множество делителей нуля кольца $R_{2}$ и $D=\{0, \alpha, \beta, \gamma\}$. Если $D^{2}=(0)$, то элементы $\{\alpha, \beta, \gamma\} \cup\{e, e+\alpha, e+\beta\}$ образуют $K_{3,3}$; получаем противоречие. Значит, $D^{2} \neq(0)$.

Если $R_{2}=J\left(R_{2}\right)$, то $R=D$, то есть $R_{2}$ - нильпотентное кольцо порядка 4, причем $R_{2}^{2} \neq(0)$. В этом случае $\Gamma\left(G F(3) \oplus R_{2}\right)$ не является планарным. Действительно, пусть $\alpha R_{2}=R_{2} \alpha=(0)$ (такой элемент в нильпотентном кольце существует). Тогда элементы $\{\alpha, \beta, \gamma\} \cup\{e, 2 e, e+\alpha\}$ образуют $K_{3,3}$; получаем противоречие. Следовательно, 
$p=2$, то есть $R=G F(2) \oplus R_{2}$. Согласно [9], $R_{2}$ изоморфно $N_{4}$, либо $N_{2,2}$. Графы делителей колец $N_{4}$ и $N_{2,2}$ равны $K_{1,2}$, то есть являются планарными. Как видно из рис. 3, граф $\Gamma\left(G F(2) \oplus N_{4}\right)$ планарен. Поскольку $\Gamma\left(G F(2) \oplus N_{4}\right)=\Gamma\left(G F(2) \oplus N_{2,2}\right)$, граф $\Gamma\left(G F(2) \oplus N_{2,2}\right)$ также планарен.

Пусть теперь $J(R) \neq R$. Кольцо $R_{2}$ не может содержать кольцо верхних треугольных матриц порядка 2 над полем $G F(2)$, так как $\Gamma\left(R_{2}\right)$ состоит ровно из трех вершин. Далее, если $R_{2}=G F(q)$, то $R$ является коммутативным кольцом с единицей, а этот случай, как мы уже отмечали, описан. Поэтому по предложению $1 R_{2} / J\left(R_{2}\right)$ является полем, причем $J\left(R_{2}\right) \neq(0)$. Так как $|D|=4$, справедливо неравенство $\left|J\left(R_{2}\right)\right| \leqslant 4$. Пусть $\left|J\left(R_{2}\right)\right| \leqslant 3$. Тогда порядок кольца $R_{2}$ равен $p^{2}$, где $p=2$, 3. Так как $\left|J\left(R_{2}\right)\right|<|D|$, кольцо $R_{2}$ не является локальным. Согласно [9], $R_{2}$ изоморфно $A_{2}$ или $A_{2}^{0}$. Справедливо равенство $\Gamma\left(A_{2}\right)=\Gamma\left(A_{2}^{0}\right)=K_{1,2}$, поэтому так же, как в случае $R_{2}=N_{4}$, можно доказать, что $\Gamma\left(G F(p) \oplus A_{2}\right)$ и $\Gamma\left(G F(p) \oplus A_{2}^{0}\right)$ планарны лишь при $p=2$. Пусть теперь $\left|J\left(R_{2}\right)\right|=4$, то есть $J\left(R_{2}\right)=D$. Поскольку $D^{2} \neq(0)$, справедливо равенство $\left|J\left(R_{2}\right)^{2}\right|=2$. Далее, $\left|R_{2} / J\left(R_{2}\right)\right| \leqslant\left|J\left(R_{2}\right)^{2}\right|$, то есть $\left|R_{2}\right|=8$. Если $R_{2}$ не содержит единицу, то все элементы кольца $R_{2}$ являются делителями нуля (см. с. 11 в [9]), чего быть не может. Следовательно, в $R_{2}$ есть единица. Таким образом, нам необходим список локальных колец порядка 8, квадрат радикала Джекобсона которых не равен нулю. Однако, как видно из [9], все такие кольца коммутативны. Поэтому и кольцо $R$ коммутативно и имеет единицу. Теорема доказана.

Автор глубоко признателен Ю. Н. Мальцеву за постановку задачи и помощь, оказанную при работе над статьей. Автор также благодарен А. А. Нечаеву и рецензенту за внимание к работе и полезные замечания.

\section{Список литературы}

1. Beck I., Coloring of commutative rings. J. Algebra (1988) 116, №1, 208-226.

2. Anderson D. F., Livingston P. S., The zero-divisor graph of a commutative ring. J. Algebra (1999) 217, №2, 434-447.

3. Akbari S., Mohammadian A., On zero-divisor graphs of finite rings. J. Algebra (2007) 314, №1, 68-184.

4. Akbari S., Maimani H. R., Yassemi S., When zero-divisor graph is planar or a complete $r$-partite graph. J. Algebra (2003) 270, №1, 169-180.

5. Belshoff R., Chapman J., Planar zero-divisor graphs. J. Algebra (2007) 316, №3, 471-480.

6. Kuzmina A. S., Maltsev Yu. N., Nilpotent finite rings with planar zero-divisor graphs. AsianEuropean J. Math. (2008) 1, №4, 565-574.

7. Харари Ф., Теория графов.. Мир, Москва, 1973.

8. Джекобсон Н., Строение колеи. ИЛ, Москва, 1961.

9. Елизаров В. П., Конечные кольца. Гелиос АРВ, Москва, 2006.

10. Ратинов В. А., Полусовершенные кольца со специальныли типами присоединенных групп, Дисс. канд. физ.-мат. наук. МГПИ, Москва, 1980.

11. Кузьмина А. С., О строении колец с планарными графами делителей нуля. Изв. Алтайского гос. ун-ma (2009) №1, 17-25.

Статья поступила 24.04.2009.

Переработанный вариант поступил 22.05.2009. 